\title{
Self-management of the health care regimen: a comparison of nurses' and cardiac patients' perceptions
}

\author{
Julia A. Dodge, Nancy K. Janz, Noreen M. Clark* \\ School of Public Health, University of Michigan, 1420 Washington Heights, Ann Arbor, MI 48109-2029, USA
}

(Received 25 February 1993; accepted 14 December 1993)

\begin{abstract}
This study compared perceptions of 129 elderly cardiac patients regarding their health status and self-management skills with corresponding assessments by outpatient nursing staff members. Nurse-patient perceptions were most congruent on general questions regarding the heart condition. Differences were most apparent regarding how aware patients were of being asked to follow a regimen for managing their heart problems; what specific components comprised the regimens the patients were to follow; and how much confidence patients had in their ability to comply with specific components of the regimen. Congruence in perceptions of nurses and patients varied by the sex of the patient. Findings suggest that careful and continuous assessment of patients' perceptions about their condition and ongoing instruction regarding specifics of the therapeutic regimen are key elements in efforts to enhance elderly patients' selfmanagement skills.
\end{abstract}

Key words: Heart disease; Self-management; Provider/patient; Interaction

\section{Introduction}

Most health behaviours directed at controlling chronic disease must be undertaken by the patient on a day to day basis. Health professionals are important providers of education and counseling to enhance patients' self-management skills. However, to be effective, efforts to teach self-management strategies must begin with an understanding of how patients see the tasks and their own abilities to carry them out. Linked to effective patient self-management behavior is confidence in one's

* Corresponding author. ability to follow the regimen prescribed by the health professional. Theoretically, if the clinician and patient share similar perceptions of the patient's situation and level of functioning, they are better able to interact regarding the goals of the therapeutic plan. The clinician is also able to provide better counseling. This study examined the extent to which perceptions of nurses about their heart patients were congruent with those of the elderly men and women who received cardiac care and education from them. The primary objective of this project was to compare assessments by nursing staff members in the clinical setting with corresponding assessments by their older cardiac 
patients regarding the patients' functional health status and ability to manage at home.

\section{Literature review}

The importance of congruence in patientprovider perceptions is summarized by Rakowski et al. [1] who state that initial misperceptions [between clinician and patient] set the stage for later complications in treatment.' Yet congruence of perceptions between older patients and their primary care providers regarding aspects of the health care regimen has received relatively little attention. The literature that is available examines the physician-patient interaction [2-4] and has tended to focus on physician communication skills and issues of patient adherence. Brody et al. [5] addressed the issue of congruence by studying the relationship between patients' levels of satisfaction with the physician encounter, the types of interventions the patients believed they had received (e.g. examinations or education/counseling), and the congruence between those interventions and the types they desired. The investigators found that patients generally claimed to receive the kind of interventions they deemed appropriate. In addition, patient satisfaction was more closely related to the physicians' attempts to address personal needs (i.e. the patients' need for information and support, rather than the need for technical care, such as laboratory tests and medication). Aspects of clinician-patient communication were explored by Helman [6] in a pilot study involving 42 adults with chronic 'psychosomatic' disorders, 12 primary care physicians, and six nurse practitioners. The degree of agreement between the provider and patient regarding the origin, significance, and effects of the patient's condition was a 'measure of the success of communication between clinician and patient at the time of the study, as well as a predictor of the success of communication in the future'. The investigator found that providers and patients appeared to use different 'explanatory models' in communicating about aspects of the patients' conditions and, therefore, were often in disagreement.

Congruence between nurses and their patients has been the focus of three studies. Tompkins [7] examined congruence with respect to nurse/patient values. Where significant differences existed in the meaningfulness attached to seven specific values, patients were more likely to attribute higher meaning than were the nurses. Evers et al. [8], in comparing self-care scores assigned by a total of 80 patients (40 institutionalized and 40 ambulatory) and their nurses, found that the scores given by the patients were higher than those assigned by the nurses. The perceived self-care abilities of $40 \mathrm{pa}$ tients compared with perceptions of the nurses assigned to visit them at home was the subject of a study by Ward-Griffin and Bramwell [9]. They found a significant positive relationship between patient and nurse perceptions of the patients' selfcare abilities, with evidence that the degree of congruence decreased with the patients' increasing age. In addition, this study found that the nurses rated the patients' health status lower than the patients rated themselves.

Limited information is available related to situations where congruence might be predicted, particularly for the elderly [10]. Rakowski et al. [1] examined congruence in perceptions of 108 elderly provider/patient pairs in a primary care clinic. Congruence varied from $29 \%$ to $69 \%$ on 14 health and treatment interview items, with patient perceptions tending to be more favorable than the perceptions of their providers. Congruence was higher for male patients and for providers who reported enjoying their interactions with older patients in the clinical setting. Occupation appeared to be a factor, with greater congruence among providers and those patients with more independent or self-directed occupations.

In summary, while research related to congruence in provider/patient perceptions is limited, findings suggest that perceptions of the health professional and patient are likely to vary related to the patients' condition and self-management capabilities. The present study was designed to focus on older adults with cardiac problems and to identify specific areas of shared perceptions between nurses and patients.

\section{Method}

\subsection{Design}

The research reported here was carried out as part of a larger study of how to enhance self- 
management skills of older adults with heart disease. The parent study was an evaluation of the effectiveness of an educational program entitled 'take PRIDE' [11]. Participants in the larger study were identified by a screening of medical records in cardiology outpatient clinics affiliated with four large hospitals in south-eastern Michigan. All persons meeting the following inclusion criteria were contacted: 60 years of age or older; diagnosed heart condition; using daily medication for the heart; seen by a physician approximately every 6 months; and living within $1 \mathrm{~h}$ of the study site. Cardiac disease was defined as any condition involving the heart itself (e.g. arrhythmia, angina, myocardial infarction, or valvular disease). Potential subjects were excluded if hypertension was their only heart-related diagnosis, since hypertension is most often asymptomatic and the intent of the program was to involve persons limited by their heart disease on a daily basis. Length of time since initial cardiac diagnosis ranged from a minimum of 6 months to 20 or more years. All patients, regardless of length of time since initial diagnosis, were a minimum of 6 months from any acute cardiac episode causing hospitalization. Clinic staff assisted in identifying individuals who would be unable to participate in telephone interviews because of significant hearing or memory loss or lack of fluency in English. Of those who met the criteria and could be contacted by telephone, $49 \%$ agreed to participate in the parent study. A total of 636 persons were recruited over a 3-year period. Persons electing not to participate were more likely to be female, older, and to have less formal education [12].

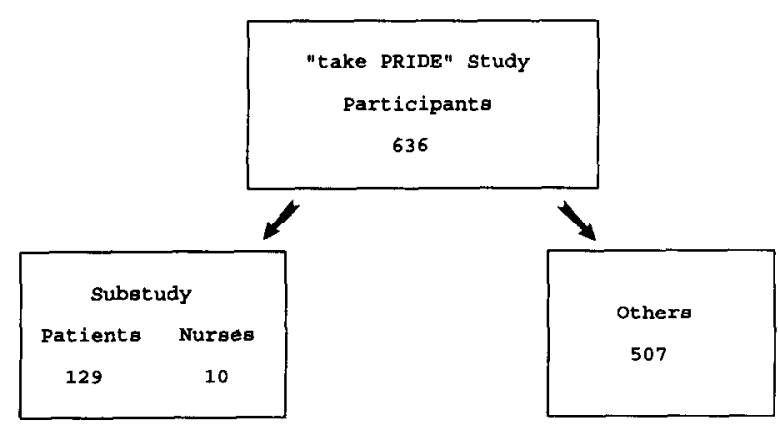

Fig. 1. Diagram of recruitment of subjects to the substudy.
The study employed telephone interviews as the primary method to collect data from patients. Interviews were conducted at baseline and 2-, 6-, $12-$, and 18-month follow-ups. Data from nurses were obtained by questionnaire at the time of the patients' scheduled outpatient visits. Clinic staff at three of the four participating hospitals were provided with a list of all subjects recruited to the parent study from their site, along with blank nursing perception questionnaires. Appointment schedules were reviewed on a weekly basis to identify returning study participants and to alert staff that a nursing questionnaire would need to be completed within $24 \mathrm{~h}$ following the visit. The research team instructed the nurses not to change their usual routine for interacting with patients. The nursing questionnaires were completed following the visit and then mailed to the research office and paired with the most recent patient telephone interview. A total of 129 pairs of nursing questionnaires and patient survey forms were completed. Ten nurses provided data on an average of 13 patients each (see Fig. 1).

The study did not attempt to document the specific circumstances surrounding the heart disease management recommendations patients were given (e.g. who made them, when, the details of instructions). In few medical settings is it possible to assess fully the precise messages the patients receive from their health care providers. Advice is usually provided by several members of the health care team, e.g. physicians, nurses, and health educators. Physicians in the study sites, as standard practice, included diet, exercise, medicinetaking, and smoking recommendations in routine counseling provided to their patients. Clinic nurses had worked with these physicians in this or a similar health care position for no less than 2 and up to 20 years. The basic management messages are routinely provided by study site physicians and reinforced by nurses. The focus of data collection was the patients' perceptions about the tasks required to manage their disease and their ability to carry out these tasks (modify diets, maintain exercise levcls, use prescription medicinc, quit smoking) compared with the nurses' perceptions. In the ideal educational situation a close match exists between patient and provider perceptions. For example, if the nurse believes that the patient 
has a high level of confidence that they can get sufficient exercise but this is an inaccurate perception, the nurse is less likely to counsel them on this aspect of management, and subsequently is less likely to be an effective educator.

\subsection{Subjects}

Patients represented in this subsample had the following sociodemographic characteristics (Table 1): $53 \%$ were male, $97 \%$ Caucasian, and $67 \%$ married. The mean age was 70.5 years. While the majority had graduated from high school, $5 \%$ had an eighth grade or less education. Most participants were retired, although $9 \%$ were still employed either part or full time.

A variety of cardiac problems were represented in the population, with $82 \%$ of the subjects having two or more cardiac diagnoses. Arrhythmia was the most frequently reported $(68 \%)$ cardiac diagnosis, followed by angina (64\%), heart attack

Table 1

Sociodemographic characteristics of patient participants (percentages)

\begin{tabular}{lccc}
\hline Characteristic & $\begin{array}{l}\text { All } \\
(n=129)\end{array}$ & $\begin{array}{l}\text { Male } \\
(n=68)\end{array}$ & $\begin{array}{l}\text { Female } \\
(n=61)\end{array}$ \\
\hline Age & & & \\
$\quad 60-69$ years & 49.6 & 47.1 & 52.5 \\
$70-79$ years & 41.1 & 45.6 & 36.1 \\
$\quad 80+$ years & 9.3 & 7.4 & 11.5 \\
Sex & & & \\
Male & 52.7 & - & - \\
Female & 47.3 & - & - \\
& & & \\
Race & & & \\
Caucasian & 96.8 & 97.1 & 96.7 \\
African-American & 1.6 & - & 3.3 \\
Other & 1.6 & 2.9 & - \\
Marital status & & & \\
Married & 67.4 & 86.8 & 45.9 \\
$\quad$ Widowed & 27.9 & 10.3 & 47.5 \\
Other & 4.7 & 2.9 & 6.6 \\
Education & & & \\
$\quad \leq$ Grade 8 & 4.7 & 7.4 & 1.6 \\
Grade 9-12 & 52.0 & 42.6 & 62.3 \\
Some college & 43.3 & 50.0 & 36.1 \\
Employment & & & \\
Employed (FT/PT) & 9.3 & 9.0 & 9.8 \\
Retired & 74.3 & 82.0 & 65.6 \\
Other & 16.4 & 9.0 & 24.6 \\
\hline & & & \\
\hline
\end{tabular}

$(56 \%)$, and high blood pressure (54\%). For $78 \%$ of these patients, the cardiovascular condition was his/her primary health problem. While the subjects were healthy enough to be ambulatory, they were all taking daily medication for their heart conditions. Among other chronic conditions being experienced by participants, arthritis was reported most often $(70 \%)$, followed by back pain $(43 \%)$, and walking problems $(36 \%)$. The mean number of comorbidities listed by the participants was 3.4.

Of the ten clinic staff participating in completing nursing assessments, six were employed in clinics affiliated with a hospital, and four were employed in private cardiologists' offices. While only nurses were recruited for participation in this substudy, written data provided by staff members subsequent to the beginning of data collection revealed that two individuals had no formal nursing training. One had received on-the-job training, while the other had completed a medical assistant program. Of the remaining eight nurses, one attended a diploma nursing program, one had an associate's degree, four bachelor's degrees, and two had master's degrees in nursing. Data from all ten staff members were included in the analysis; findings regarding the relationship of educational level to patient/provider congruence will be presented in the results section.

\subsection{Instruments}

The nursing perception questionnaire was specifically designed to mirror questions asked of participants on their periodic telephone interviews. The questions had been pretested with older adults and reviewed by the nurse providers to ensure that wording and response categories were clear. For each nurse-patient pair, data were collected for a single time point. While $50 \%$ of the completed nursing questionnaires coincided with the patient's 12-month follow-up interview in the larger study, 2 -month $(2 \%)$, 6-month $(13 \%)$, and 18 -month $(35 \%)$ data for some patients were utilized alternatively in order to minimize the length of time between the patient's telephone interview and the outpatient visit when the nursing questionnaire was completed.

The questionnaires completed by both the clinic staff and the patient contained ten global items 
addressing perceptions of health status, social comparison with peers, awareness of specific components of the health regimen, and overall selfefficacy regarding heart self-management. Items addressing perceptions of health status included the seriousness of the patient's heart condition, the patient's level of worry regarding the heart condition, the extent to which the heart condition interfered with the patient's life, the degree of difficulty in functioning faced by the patient because of the heart condition and overall physical functioning. Social comparison items assessed perceptions of the patient's health compared with others of the same age and the patient's ability to manage compared with others with the same heart problem. Self-efficacy items tapped the patient's overall confidence to manage the heart recommendations and the general level of self-confidence of the patient. Perceptions of the patient's state of health since the last clinic visit were also assessed. Nine questions assessed specific components of the self-management plan the patient was to follow at home (i.e. medicine-taking, diet, exercise, and smoking recommendations), both in terms of how aware each was of what the patient had been asked to do by a health professional, and the patient's levels of confidence of complying with the recommendations. All questions were closedend with four or five predetermined response categories. Responses to questions regarding perceptions of self-confidence were rated on a scale from 1 to 10 , where 1 was 'not at all confident' and 10 was 'very confident'. All questions were worded identically for nurses and patients, except for a self-reference ('you' or 'your') in the patient interview and reference to 'the patient' in the nurse's questionnaire.

Chi-square and Cramer's phi statistics were first used to determine the degree of correspondence or congruence between patient and nurse perceptions. A cross-tabulation of each group's responses on individual questions was completed so that congruent pairs fell on the diagonal. Responses were then collapsed from four or five categories into three categories (nurse rated the item more favorably, nurse and patient rated the item the same, patient rated the item more favorably). Percentages of complete congruence were then calculated for each of the questions, as well as the frequencies with which the patient's or nurse's responses were more favorable.

\section{Results}

\subsection{Global perceptions of the heart condition}

On the 19 specific questions asked of both patients and their nurse providers, there were no significant differences in perception reported in $58 \%$ of the responses, a figure supported in the literature regarding patient-provider congruence [1]. In several areas, however, there were significant differences. Table 2 presents nine of the individual questions related to general perceptions of the heart condition; congruence varied from $25.6 \%$ to $58.6 \%$.

Nurses were significantly more likely to rate patients' heart conditions as less serious $(P \leq 0.01)$ than the patients' own ratings. Patient physical functioning was rated significantly lower $(P \leq 0.01)$ by the nurses than by the patients, as was overall patient self-confidence $(P=0.04)$. In addition, patients were significantly more likely than nurses $(P \leq 0.01)$ to perceive their health as better compared with peers of the same age. No significant differences were found with regard to worry about the heart condition, the impact of the heart condition on daily life, the degree of difficulty in managing due to the heart condition, perceptions of patient management of the heart problem compared with others with the same condition, and health since the last clinic visit. Finally, no significant differences in mean overall self-confidence scores between nurses and their patients were found.

To determine if the age or sex of the patient made a difference in congruence between patient and nurse responses, we analysed the ten questions related to general perceptions of the heart condition according to age (60-69 years vs. 70 years and older). Nurses were significantly more likely to rate physical functioning as lower, irrespective of patient age. However, with respect to perceived seriousness of the heart condition, the discrepancy between nurse and patient responses occurred only when the patients were younger, i.e. 60-69 years, with nurses viewing the heart condition as less seri- 
Table 2

Rates of congruence and noncongruence among patients and nurses on general perceptions of the heart condition (percentages)

\begin{tabular}{llll}
\hline General health questions & Congruence & \multicolumn{2}{l}{ Non-congruence } \\
\cline { 3 - 4 } & & $\begin{array}{l}\text { Patient more } \\
\text { favorable }\end{array}$ & $\begin{array}{l}\text { Nurse more } \\
\text { favorable }\end{array}$ \\
\hline Seriousness of heart condition** & 28.7 & 34.9 & 36.4 \\
Worry regarding heart condition & 35.7 & 47.2 & 17.1 \\
Impact of heart condition & 25.6 & 45.0 & 29.4 \\
Difficulty due to heart condition & 28.1 & 50.0 & 21.9 \\
Overall physical function** & 48.1 & 30.2 & 21.7 \\
Health compared with age peers** & 36.3 & 49.2 & 14.5 \\
Manage compared with others with same & 39.4 & 44.9 & 15.7 \\
$\quad$ heart condition & 35.7 & 37.2 & 27.1 \\
Overall self-confidence & 58.6 & 20.3 & 21.1 \\
Health since last visit & & & \\
\hline
\end{tabular}

$* P \leq 0.05$ for significance between patient and nurse responses; $\chi^{2}$ test.

${ }^{* *} P \leq 0.01$ for significance between patient and nurse responses; $\chi^{2}$ test.

ous. With this younger age group, a significant difference also emerged regarding the perceived degree of interference of the heart condition with daily functioning. Nurses perceived a greater degree of interference than did the patients. Regarding gender differences (Table 3), males were significantly more likely than nurses $(P \leq 0.01)$ to perceive their health as better compared with peers of the same age.

A finding emerged with respect to the congruence between nurses and their female patients in perceptions of the seriousness of the heart disease. Nurses were significantly more likely to rate the heart condition as more serious $(P=$ 0.01 ). In particular, over $15 \%$ of female patients rated their heart condition as 'not at all serious.' In no case did the nurse make such a rating.

\subsection{Self-management recommendations}

Regarding self-management recommendations that patients were asked to follow, overall, and with the younger cohort (60-69 years), nurses

Table 3

Comparison of patient and nurse responses on general perceptions of the heart condition, by sex

\begin{tabular}{|c|c|c|c|c|c|c|}
\hline \multirow[t]{2}{*}{ General health questions } & \multicolumn{3}{|c|}{ Male $(n=68)$} & \multicolumn{3}{|c|}{ Female $(n=61)$} \\
\hline & $x^{2}$ & $P$-value & Interpretation & $\chi^{2}$ & $P$-value & Interpretation \\
\hline Seriousness of heart condition & 17.55 & 0.35 & & 44.57 & $0.001^{* *}$ & $\begin{array}{l}\text { Nurses rate as more } \\
\text { serious }\end{array}$ \\
\hline Worry regarding heart condition & 12.74 & 0.17 & & 11.21 & 0.26 & \\
\hline Impact of heart condition & 20.34 & 0.21 & & 16.65 & 0.41 & \\
\hline Difficulty due to heart condition & 15.89 & 0.46 & & 23.84 & 0.09 & \\
\hline Overall physical function & 35.07 & $0.004^{* *}$ & Nurses rate lower & 30.82 & $0.01^{* *}$ & Nurses rate lower \\
\hline Health compared with age peers & 40.62 & $0.001^{* *}$ & Nurses rate lower & 10.81 & 0.29 & \\
\hline $\begin{array}{l}\text { Manage compared with others } \\
\text { with same heart condition }\end{array}$ & 4.67 & 0.59 & & 13.18 & 0.11 & \\
\hline Overall self-confidence & 10.53 & 0.31 & & 11.65 & 0.23 & \\
\hline Health since last visit & 6.93 & 0.14 & & 2.38 & 0.67 & \\
\hline
\end{tabular}

${ }^{*} P \leq 0.05$ for significance between patient and nurse responses; $x^{2}$ test.

${ }^{* *} P \leq 0.01$ for significance between patient and nurse responses; $\chi^{2}$ test. 
were significantly more likely than their patients to state that a special diet $(P \leq 0.01)$ or exercise program $(P \leq 0.01)$ had been recommended by the health professional. No differences were found with respect to perceptions about whether or not heart medications had been prescribed. No differences were found between the perceptions of nurses and their older patients (70 years and over) regarding whether medication, diet, and exercise prescriptions had been given by the health care provider. When the data were examined to determine if these perceptions differed by the sex of the patient, nurses were significantly more likely than their male patients to state that an exercise program had been prescribed $(P \leq 0.01)$ and significantly more likely than their female patients to state that a special diet had been recommended $(P \leq 0.01)$. Because only three of the 129 patients reported that they were currently cigarette smokers, statistical tests could not be computed for the questions related to this aspect of self-management.

Table 4 presents the mean ratings of patient perceived self-confidence in managing specific aspects of the heart problem. Nurses consistently gave lower ratings of patients' confidence to follow their medication $(P \leq 0.01)$ and diet $(P=0.03)$ regimens than did the patients themselves. No significant differences were found with regard to perceived self-efficacy to follow an exercise regimen. Both nurses and patients felt

Table 4

Patient and nurse perceptions of self-confidence ${ }^{\mathrm{A}}$ to follow heart regimen components

\begin{tabular}{lll}
\hline Variable & $\begin{array}{l}\text { Patient } \\
(n=129) \\
(\mathrm{M})\end{array}$ & $\begin{array}{l}\text { Nurse } \\
(n=10) \\
(\mathrm{M})\end{array}$ \\
\hline $\begin{array}{ll}\text { Rating of confidence } \\
\text { Medication }\end{array}$ & 9.6 & $8.8^{* *}$ \\
Diet & 8.1 & $7.6^{*}$ \\
Exercise & 8.8 & 8.1
\end{tabular}

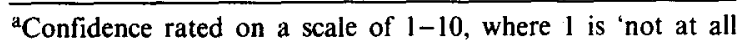
confident' and 10 is 'very confident.'

${ }^{*} P \leq 0.05$ for significance between patient and nurse responses; $t$ test.

${ }^{* * P} \leq 0.01$ for significance between patient and nurse responses; $t$ test. least optimistic regarding the patients' ability to follow a diet regimen.

When differences in self-efficacy perceptions were examined by gender (Table 5), nurses' ratings of patients' ability to follow their prescribed medication regimens were significantly lower than ratings given by both male $(P \leq 0.01)$ and female $(P \leq 0.01)$ patients. Patients' confidence to follow diet recommendations was rated significantly lower by the nurses for their female patients $(P=0.03)$, while the nurses rated confidence to follow an exercise program significantly lower for male patients $(P=0.04)$. It is interesting to note that when participants were asked to rate their own levels of confidence to follow an exercise regimen, the mean score for males was $9.3 \mathrm{com}$ pared with 7.7 for female patients, indicating that females in this sample had considerably less confidence in the area of exercise. Across all age groups nurses consistently rated the patients' perceived level of self-efficacy to follow the medication regimen as significantly lower than that of the patient $(P=0.01)$. Self-efficacy to follow the diet regimen was rated significantly lower by nurses as compared with younger (60-61 years) patients $(P=0.02)$.

\subsection{Characteristics of patients and providers}

While some initial analyses were done to determine if patient sociodemographic characteristics

Table 5

Patient and nurse perceptions of self-confidence ${ }^{\mathrm{a}}$ to follow heart regimen components, analysed by sex of client

\begin{tabular}{|c|c|c|c|c|}
\hline \multirow[t]{2}{*}{ Variable } & \multicolumn{2}{|l|}{ Male } & \multicolumn{2}{|l|}{ Female } \\
\hline & $\begin{array}{l}\text { Patient } \\
\text { (M) }\end{array}$ & $\begin{array}{l}\text { Nurse } \\
\text { (M) }\end{array}$ & $\begin{array}{l}\text { Patient } \\
\text { (M) }\end{array}$ & $\begin{array}{l}\text { Nurse } \\
\text { (M) }\end{array}$ \\
\hline \multicolumn{5}{|c|}{ Rating of confidence } \\
\hline Medication & 9.5 & $8.9^{* *}$ & 9.6 & $8.6^{* *}$ \\
\hline Diet & 7.9 & 7.6 & 8.3 & $7.6^{*}$ \\
\hline Exercise & 9.3 & $8.5^{*}$ & 7.7 & 7.4 \\
\hline
\end{tabular}

${ }^{a}$ Confidence rated on a scale of $1-10$, where 1 is 'not at all confident' and 10 is 'very confident.'

${ }^{*} P \leq 0.05$ for significance between patient and nurse responses; $t$ test.

${ }^{* *} P \leq 0.01$ for significance between patient and nurse responses; $t$ test. 
other than gender and age (e.g. race, marital and employment status) affected congruence, the sample size was not large enough to allow any definitive conclusions.

In terms of characteristics of the clinic staff, educational level was not a significant factor in determining the degree of correspondence between responses. Since the clinic staff were all fcmalc, this research could not determine if gender of the provider was a factor. Congruence between patient and nurse responses was greater when the nurse knew the patient for 1 year or less compared with greater than 1 year. This finding was not related to the age of the patient. As one might expect, the shorter the interval between the patients' completing the telephone interview and the nurses' completing the questionnaire in the office, the greater the degree of correspondence. To determine the effect, if any, of the window of time between completion of interviews by nurses and patients, a separate analysis was conducted of responses within and outside a 1-month window. For 53 patients the window was 1 month or less, for 67 patients 1-3 months, and for 9 patients more than 3 months. Significant differences between groups were found for several variables according to the window of time between interviews. The bigger the window of time, the less the congruence between nurses and patients on the seriousness of the heart problem (the nurses deemed it more severe), the level of the patient's physical ability (the nurses said it was lower) and the perception of the patient's health compared with other people of approximately the same age (the nurses rated it as worse). The nurses also believed the patients to have less confidence to manage their heart conditions when the window between patient and nurse responses was increased. While the level of patient-nurse congruence increased as time between the patient and nurse data collection decreased, several areas important to education and counseling of patients remained different, even within a 1 -month window. Nurses more often reported that their patients had been asked to follow a special diet or exercise program while patients disputed the fact. Nurses also rated patient confidence to follow medication recommendations lower than did the patients themselves.

\section{Discussion}

The findings from this study suggest ways in which health care providers can modify their practices to address more specifically the needs of older adults with heart conditions (Table 6). The generalizability of the findings is somewhat limited by the relatively high level of education of the study participants. The data are, in large measure, reassuring in that they suggest that nurses generally share their older cardiac patients' perceptions of overall health status and self-management skills. They support previous estimates of patientprovider congruence in the literature which range from $50 \%$ to $70 \%$ [1].

However, one area in which significant differences were detected involved seriousness of the heart condition. Across the entire sample, nurses tended to view the patients' heart conditions as less serious. This may reflect the nurses' broadbased experience with heart patients as opposed to the patient's personal 'sample of one' assessment. An interesting paradox occurs with the finding that $15 \%$ of female patients rated their heart conditions as 'not at all serious' while no nurse made such a rating. The Health Belief Model, a major organizing framework for explaining and

Table 6

Implications for practice

1. Nurse/client perceptions were generally congruent. Differences were most apparent regarding specific components of the heart self-management recommendations, indicating that careful and continuous assessment of the regimen is warranted.

2. Congruence of the nurse/client perceptions varied by the sex of the client, suggesting that providers need to assess and tailor interactions with sensitivity to possible gender differences.

3. Overall, clients have high levels of confidence in their ability to follow health recommendations. Reinforcement of progress will assist clients to maintain higher levels of confidence.

4. Males are least confident regarding their ability to follow a diet regimen: females are least confident regarding their exercise regimen. Education and support regarding aspects of the heart recommendations should reflect these gender differences. 
predicting acceptance of health and medical recommendations, hypothesizes that healthrelated behaviors depend upon a person's perceptions regarding four dimensions (susceptibility, severity, benefits, and barriers) $[13,14]$. If an individual does not perceive a condition as serious (i.e. low threat), the Health Belief Model would suggest that he/she would be less likely to see the need for following the recommended actions. By directly assessing and discussing perceptions of seriousness of the heart disease with patients, particularly women, clinical staff will have a context within which to identify those patients whose perceptions are unrealistic (i.e. those who feel less vulnerable and are less likely to take needed actions to deal with their disease).

The nurses in our sample consistently differed from their patients regarding perceptions of physical functioning, rating the patients' functioning as lower, a finding consistent with previous studies $[15,16]$. The noted discrepancy may be a mixture of patients' overestimating the amount of exercise they are getting on a regular basis, and the nurses' lack of awareness of some patients' levels of routine exercise. More careful assessment is warranted so that nurses can better educate patients about approaches for adequate exercise.

As with physical functioning, patients, particularly men, were significantly more likely to rate their overall health compared with that of their peers as higher than nurses rated it. Ward-Griffith and Bramwell [9] reported a similar finding and suggested that nurses and patients may be operating under different definitions of health. Traditional nursing education and, in fact, the present health care system, operate under a medical illness-oriented model where the focus is on illness and resulting limitations and deficiencies. The patient, on the other hand, may view health more broadly. Rather than focusing on specific illness conditions, the patient may view health in terms of functional ability, independence, and other factors which relate to a personal assessment of life quality.

Study findings suggest the importance of nurses' routinely assessing older patients' understanding of each component of their self-management plan and being sensitive to possible gender differences.
While in this research nurses and patients agreed that medications had been ordered, they differed significantly in their perceptions of whether a special diet or exercise had been prescribed. When a difference was found, it was the patient who did not recall being asked to follow a special diet or exercise program. There is need to reassess continually the recommendations made to patients for managing their heart problems at home and to make self-management the basis of counseling. In the clinical setting, assessment can be done with a minimum expenditure of additional time. A few simple questions could be asked, e.g. 'What exercise program have you been asked to follow?', followed by 'How have you been doing with that program since your last visit?'. In addition to providing verbal instructions to reinforce the patient's behavior, nurses might also provide written information and ask the patient to repeat verbally instructions before leaving the clinic.

Patients in this study rated their self-efficacy to follow all components of their self-management plan higher than did the nurses. These ratings by the patients suggest that overall they do, in fact, have confidence that they can carry out the expected behaviors. However, as noted above, nurses may want to focus especially on enhancing their female patients' efficacy regarding exercise as these levels were, in general, lower than among male patients. Verbal persuasion by health providers is one source for developing and enhancing confidence. Therefore, reinforcement by nurses of even small steps in adherence will assist the patients to maintain the higher levels of efficacy generally observed in this study. Also, research has shown that women are more likely than men to live alone, to experience comorbidities, and to report particular difficulty with physical activity and symptom management [17]. A special effort is needed to introduce innovative ways by which women can enhance physical functioning and more successfully follow the exercise recommendations of their physicians within the context of their current living situations.

Finally, the data suggest one subgroup who might require more careful nursing assessment with regard to both general functioning and perceptions regarding specific aspects of their 
heart regimen. Significant differences in perceptions were noted between nurses and the younger cohort in this study, specifically those aged 60-69 years. While the reasons for this difference are not clear, this decade is one in which patients are frequently being diagnosed with heart conditions for the first time. In contrast to older patients who may have had time to adjust to their diagnoses and heart self-management recommendations, younger patients are likely to vary more in their perceptions and understanding and, as a result, might benefit from more careful assessment and teaching.

In summary, the purpose of this study was to identify discrepancies in provider and patient perceptions of the medical regimen. Findings support results of previous studies and suggest that communication between health care provider and patient may be more difficult to achieve and maintain over time than one might naturally assume. The results highlight the need for careful and ongoing assessment by clinicians. Clinical staff are central in the delivery of health education and central to effective counseling is assessment and reinforcement of patients' positive self-management skills. If the health professional does not recognize lack of congruence between his/her perceptions and those of the patient, then the counseling strategies used are less likely to be effective. This study underscores the need to work closely with patients and to develop collaboratively with them strategies for enhancing their health-related quality of life.

\section{Acknowledgments}

This study was funded by The Henry J. Kaiser Family Foundation; The National Heart, Lung, and Blood Institute, National Institutes of Health; and The American Heart Association of Michigan.

\section{References}

I Rakowski W, Hickey T, Dengiz AN: Congruence of health and treatment perceptions among older patients and providers of primary care. Int $\mathbf{J}$ Aging Hum Dev 1987; 25: 63-77.
2 Anderson LA, Sharpe PA: Improving patient and provider communication: a synthesis and review of communication interventions. Patient Educ Couns 1991; 17: 99-134.

3 Friedman HS, DiMatteo MR: Patient-physician interactions. In: Shumaker SA, Schron EB, Okene JK eds. Handbook of Health Behavior Change. New York: Springer-Verlag, 1990, pp. 84-101.

4 Walsh JME, McPhee SJ: A systems model of clinical preventive care: an analysis of factors influencing patient and physician. Health Educ Q 1992; 19: 157-175.

5 Brody DS, Miller SM, Lerman CE, Smith DG, Lazaro CG, Blum MJ: The relationship between patients' satisfaction with their physicians and perceptions about interventions they desired and received. Med Care 1989; 27: 1027-1035.

6 Helman CG: Communication in primary care: the role of patient and practitioner explanatory models. Soc Sci Med 1985; 20: 923-931.

7 Tompkins ES: Nurse/client values congruence. West J Nurs Res 1992; 14: 225-236.

8 Evers G, Isenberg M, Philipsen H, Brouns G, Halfens R, Smeets H: The 'appraisal of self-care agency' ASA-Scale: research program to test reliability and validity. In: Stinson $\mathrm{S}$ ed. New Frontiers in Nursing Research: International Nursing Research Proceedings. Edmonton, Canada: Faculty of Nursing, 1986.

9 Ward-Griffin C, Bramwell L: The congruence of elderly client and nurse perceptions of the clients' self-care agency. J Adv Nurs 1990; 15: 1070-1077.

10 Haug MR, Ory MG: Issues in elderly patient-provider interactions. Res Aging 1987; 9: 3-44.

11 Clark NM, Janz NK, Becker MH et al.: Impact of selfmanagement education on the functional health status of older adults with heart disease. Gerontologist 1992; 32: 438-443.

12 Dodge JA, Clark NM, Janz NK, Liang J, Schork MA: Nonparticipation of older adults in a heart disease selfmanagement project: factors influencing involvement. Res Aging 1993; 15: 220-237.

13 Rosenstock IM: Historical origins of the health belief model. Health Educ Monogr 1974; 2: 328-335.

14 Becker MH, Haefner DP, Kasl SV: Selected psychosocial models and correlates of individual health-related behaviors. Med Care 1977; 15 (5 Suppl): 27-46.

15 Elam JT, Graney MJ, Beaver T, El Derwi D, Applegate WB, Miller ST: Cmparison of subjective ratings of function with observed functional ability of frail older persons. Am J Public Health 1991; 81: 1127-1130.

16 Rubenstein LZ, Schairer C, Wieland GD, Kane R: Systematic biases in functional status assessment of elderly adults: effects of different data sources. J Gerontol 1984; 39: 686 691.

17 Sharpe PA, Clark NM, Janz NK: Differences in the impact and management of heart disease between older women and men. Women Health 1991; 17: 25-43. 